\title{
Better Gap-Hamming Lower Bounds via Better Round Elimination
}

\author{
Joshua Brody ${ }^{1, \star}$, Amit Chakrabarti ${ }^{1, \star \star}$, Oded Regev ${ }^{2, \star \star \star}$, \\ Thomas Vidick ${ }^{3, \dagger}$, and Ronald de Wolf ${ }^{4, \ddagger}$ \\ 1 Department of Computer Science, Dartmouth College, Hanover, NH 03755 \\ 2 Blavatnik School of Computer Science, Tel Aviv University, Tel Aviv 69978, Israel \\ ${ }^{3}$ Department of Computer Science, UC Berkeley \\ ${ }^{4}$ CWI Amsterdam
}

\begin{abstract}
Gap Hamming Distance is a well-studied problem in communication complexity, in which Alice and Bob have to decide whether the Hamming distance between their respective $n$-bit inputs is less than $n / 2-\sqrt{n}$ or greater than $n / 2+\sqrt{n}$. We show that every $k$-round bounded-error communication protocol for this problem sends a message of at least $\Omega\left(n /\left(k^{2} \log k\right)\right)$ bits. This lower bound has an exponentially better dependence on the number of rounds than the previous best bound, due to Brody and Chakrabarti. Our communication lower bound implies strong space lower bounds on algorithms for a number of data stream computations, such as approximating the number of distinct elements in a stream.
\end{abstract}

Keywords: Communication Complexity, Gap Hamming Distance, Round Elimination, Measure Concentration.

\section{Introduction}

\subsection{The Communication Complexity of the Gap Hamming Distance Problem}

Communication complexity studies the communication requirements of distributed computing. In its simplest and best-studied setting, two players, Alice and Bob, receive inputs $x$ and $y$, respectively, and are required to compute some function $f(x, y)$. Clearly, for most functions $f$, the two players need to communicate to solve this problem. The basic question of communication complexity is the minimal amount of communication

* Supported in part by NSF Grant CCF-0448277. Part of this work was done while the author was visiting CWI and Tel Aviv University.


Fellowship.

${ }^{\star \star \star}$ Supported by the Israel Science Foundation, by the European Commission under the Integrated Project QAP funded by the IST directorate as Contract Number 015848, by the Wolfson Family Charitable Trust, and by a European Research Council (ERC) Starting Grant.

$\dagger$ Supported by ARO Grant W911NF-09-1-0440 and NSF Grant CCF-0905626. Part of this work was done while the author was visiting CWI and Tel Aviv University.

¥ Supported by a Vidi grant from Netherlands Organization for Scientific Research (NWO). 
needed. By abstracting away from the resources of local computation time and space, communication complexity gives us a bare-bones but elegant model of distributed computing. It is interesting for its own sake but is also useful as one of our main sources of lower bounds in many other models of computation, including data structures, circuits, Turing machines, VLSI, and streaming algorithms. The basic results are excellently covered in the book of Kushilevitz and Nisan [12], but many additional fundamental results have appeared since its publication in 1997.

One of the few basic problems whose randomized communication complexity is not yet well-understood, is the Gap Hamming Distance (GHD) problem, defined as follows.

GHD: Alice receives input $x \in\{0,1\}^{n}$ and Bob receives input $y \in\{0,1\}^{n}$, with the promise that $|\Delta(x, y)-n / 2| \geq \sqrt{n}$, where $\Delta$ denotes the Hamming distance. Decide whether $\Delta(x, y)<n / 2$ or $\Delta(x, y)>n / 2$.

Mind the gap between $n / 2-\sqrt{n}$ and $n / 2+\sqrt{n}$, which is what makes this problem interesting and useful. Indeed, the communication complexity of the gapless version, where there is no promise on the inputs, can easily be seen to be linear (for instance by a reduction from disjointness). The gap makes the problem easier, and the question is how it affects the communication complexity: does it remain linear? A gap size of $\Theta(\sqrt{n})$ is the natural choice - a $\Theta(1)$ fraction of the inputs lie inside the promise area for this gap size, and as we'll see below, it is precisely this choice of gap size that has strong implications for streaming algorithms lower bounds. Moreover, understanding the complexity of the $\sqrt{n}$-gap version can be shown to imply a complete understanding of the GHD problem for all gaps.

Randomized protocols for GHD and more general problems can be obtained by sampling. Suppose for instance that it is promised that either $\Delta(x, y) \leq(1 / 2-\gamma) n$ or $\Delta(x, y) \geq(1 / 2+\gamma) n$. Choosing an index $i \in[n]$ at random, the predicate $\left[x_{i} \neq y_{i}\right]$ is a coin flip with heads probability $\leq 1 / 2-\gamma$ in the first case and $\geq 1 / 2+\gamma$ in the second. It is known that flipping such a coin $\Theta\left(1 / \gamma^{2}\right)$ times suffices to distinguish these two cases with probability at least $2 / 3$. Hence if we use shared randomness to choose $\Theta\left(1 / \gamma^{2}\right)$ indices, we obtain a one-round bounded-error protocol with communication $\Theta\left(1 / \gamma^{2}\right)$ bits. In particular, for GHD (where $\gamma=1 / \sqrt{n}$ ), the communication is $\Theta(n)$ bits, which is no better than the trivial upper bound of $n$ when Alice just sends $x$ to Bob.

What about lower bounds? Indyk and Woodruff [10] managed to prove a linear lower bound for the case of one-round protocols for GHD, where there is only one message from Alice to Bob (see also [20[11]). However, going beyond one-round bounds turned out to be quite a difficult problem. Recently, Brody and Chakrabarti [5] obtained linear lower bounds for all constant-round protocols:

Theorem 1. [5] Every k-round bounded-error protocol for GHD sends a message of length $\frac{n}{2^{O\left(k^{2}\right)}}$.

In fact their bound is significant as long as the number of rounds is $k \leq c_{0} \sqrt{\log n}$, for a universal constant $c_{0}$. Regarding lower bounds that hold irrespective of the number of rounds, an easy reduction gives an $\Omega(\sqrt{n})$ lower bound (which is folklore): take an instance of the gapless version of the problem on $x, y \in\{0,1\}^{\sqrt{n}}$ and "repeat" $x$ and $y \sqrt{n}$ times each. This blows up the gap from 1 to $\sqrt{n}$, giving an instance of GHD on 
$n$ bits. Solving this $n$-bit instance of GHD solves the $\sqrt{n}$-bit instance of the gapless problem. Since we have a linear lower bound for the latter, we obtain a general $\Omega(\sqrt{n})$ bound for GHD 1

\subsection{Our Results}

Our main result is an improvement of the bound of Brody and Chakrabarti, with an exponentially better dependence on the number of rounds:

Theorem 2. Every k-round bounded-error protocol for GHD sends a message of length $\Omega\left(\frac{n}{k^{2} \log k}\right)$.

In fact we get a bound for the more general problem of distinguishing distance $\Delta(x, y) \leq$ $(1 / 2-\gamma) n$ from $\Delta(x, y) \geq(1 / 2+\gamma) n$, as long as $\gamma=\Omega(1 / \sqrt{n})$ : for this problem every $k$-round protocol sends a message of $\Omega\left(\frac{1}{k^{2} \log k} \frac{1}{\gamma^{2}}\right)$ bits.

Like the result of [5], our lower bound deteriorates with the number of rounds. Also like their result, our proof is based on round elimination, an important framework for proving communication lower bounds. Our proof contains an important insight into this framework that we now explain.

A communication problem usually involves a number of parameters, such as the input size, an error bound, and in our case the gap size. The round elimination framework consists of showing that a $k$-round protocol solving a communication problem for a class $\mathcal{C}$ of parameters can be turned into a $(k-1)$-round protocol for an easier class $\mathcal{C}^{\prime}$, provided the message communicated in the first round is short. This fact is then applied repeatedly to obtain a 0-round protocol (say), for some nontrivial class of instances. The resulting contradiction can then be recast as a communication lower bound. Historically, the easier class $\mathcal{C}^{\prime}$ has contained smaller input length $\sqrt{2}$ than those in $\mathcal{C}$.

In contrast to previous applications of round elimination, we manage to avoid shrinking the input length: the simplification will instead come from a slight deterioration in the error parameter. Here is how this works. If Alice's first message is short, then there is a specific message and a large set $A$ of inputs on which Alice would have sent that message. Roughly speaking, we can use the largeness of $A$ to show that almost any input $\tilde{x}$ for Alice is close to $A$ in Hamming distance. Therefore, Alice can "move" $\tilde{x}$ to its nearest neighbor, $x$, in $A$ : this makes her first message redundant, as it is constant for all inputs $x \in A$. Since $x$ and $\tilde{x}$ have small Hamming distance, it is likely that both pairs

\footnotetext{
${ }^{1}$ In fact the same proof lower-bounds the quantum communication complexity; a linear quantum lower bound for the gapless version follows easily from Razborov's work [18] and the observation that $\Delta(x, y)=|x|+|y|-2|x \wedge y|$. However, as Brody and Chakrabarti observed, in the quantum case this $\sqrt{n}$ lower bound is essentially tight: there is a bounded-error quantum protocol, based on a well-known quantum algorithm for approximate counting, that communicates $O(\sqrt{n} \log n)$ qubits. This also implies that lower bound techniques which apply to quantum protocols, such as discrepancy, factorization norms [15]13], and the pattern matrix method [19], cannot prove better bounds for classical protocols.

${ }^{2}$ In fact, $\mathcal{C}$ and $\mathcal{C}^{\prime}$ are often designed such that an instance in $\mathcal{C}$ is a "direct sum" of several independent instances in $\mathcal{C}^{\prime}$.
} 
$(\tilde{x}, y)$ and $(x, y)$ are on the same side of the gap, i.e. have the same GHD value. Hence the correctness of the new protocol, which is one round shorter, is only mildly affected by the move. Eliminating all $k$ rounds in this manner, while carefully keeping track of the accumulating errors, yields a lower bound of $\Omega\left(n /\left(k^{4} \log ^{2} k\right)\right)$ on the maximum message length of any $k$-round bounded-error protocol for GHD.

Notice that this lower bound is slightly weaker than the above-stated bound of $\Omega\left(n /\left(k^{2} \log k\right)\right)$. To obtain the stronger bound, we leave the purely combinatorial setting and analyze a version of GHD on the unit sphere 3 Alice's input is now a unit vector $x \in \mathbb{R}^{n}$ and Bob's input is a unit vector $y \in \mathbb{R}^{n}$, with the promise that either $x \cdot y \geq 1 / \sqrt{n}$ or $x \cdot y \leq-1 / \sqrt{n}$ (as we show below in Section 2, this version and the Boolean one are essentially equivalent in terms of communication complexity). Alice's input is now close to the large, constant-message set $A$ in Euclidean distance. The rest of the proof is as outlined above, but the final bound is stronger than in the combinatorial proof for reasons that are discussed in Section 2.2. Although this proof uses arguments from high-dimensional geometry, such as measure concentration, it arguably remains conceptually simpler than the one in [5].

Related work. The round elimination technique was first formalized in Miltersen et al. [17] and dates back even further, at least to Ajtai's lower bound for predecessor data structures [1]. For us, the most relevant previous use of this technique is in the result by Brody and Chakrabarti [5], where a weaker lower bound is proved on GHD.

Their proof, as ours, identifies a large subset $A$ of inputs on which Alice sends the same message. The "largeness" of $A$ is used to identify a suitable subset of $(n / 3)$ coordinates such that Alice can "lift" any $(n / 3)$-bit input $\tilde{x}$, defined on these coordinates, to some $n$-bit input $x \in A$. In the resulting protocol for $(n / 3)$-bit inputs, the first message is now constant, hence redundant, and can be eliminated.

The input size thus shrinks from $n$ to $n / 3$ in one round elimination step. As a result of this constant-factor shrinkage, the Brody-Chakrabarti final lower bound necessarily decays exponentially with the number of rounds. Our proof crucially avoids this shrinkage of input size by instead considering the geometry of the set $A$, and exploiting the natural invariance of the GHD predicate to small perturbations of the inputs.

Remark. After we obtained our results, a subset of the authors independently proved an optimal $\Omega(n)$ lower bound, independent of the number of rounds [6]. However, the techniques they introduce are completely different, and rather involved. In contrast, our result, through its relatively simple and elegant proof, should be of independent interest to the community.

\subsection{Applications to Streaming}

The introduction of gapped versions of the Hamming distance problem by Indyk and Woodruff [10] was motivated by the streaming model of computation, in particular the

\footnotetext{
${ }^{3}$ The idea of going to the unit sphere was also used by Jayram et al. [11] for a simplified one-round lower bound. As we will see in Section 2, doing so is perhaps even more natural than working with the combinatorial version; in particular it is then easy to make GHD into a dimension-independent problem.
} 
problem of approximating the number of distinct elements in a data stream. For many data stream problems, including the distinct elements problem, the goal is to output a multiplicative approximation of some real-valued quantity. Usually, both randomization and approximation are required. When both are allowed, there are often remarkably space-efficient solutions.

As Indyk and Woodruff showed, communication lower bounds for the Gap Hamming Distance problem imply space lower bounds on algorithms that output the number of distinct elements in a data stream up to a multiplicative approximation factor $1 \pm \gamma$. The reduction from GHD works as follows. Alice converts her $n$-bit string $x=x_{1} x_{2} \cdots x_{n}$ into a stream of tuples $\sigma=\left\langle\left(1, x_{1}\right),\left(2, x_{2}\right), \ldots,\left(n, x_{n}\right)\right\rangle$. Bob converts $y$ into $\tau=$ $\left\langle\left(1, y_{1}\right),\left(2, y_{2}\right), \ldots,\left(n, y_{n}\right)\right\rangle$ in a similar fashion. Using a streaming algorithm for the distinct elements problem, Alice processes $\sigma$ and sends the memory contents to Bob, who then processes $\tau$ starting from where Alice left off. In this way, they estimate the number of distinct elements in $\sigma \circ \tau$. Note that each element in $\sigma$ is unique, and that elements in $\tau$ are distinct from elements in $\sigma$ precisely when $x_{i} \neq y_{i}$. Hence, an accurate approximation $(\gamma=\Omega(1 / \sqrt{n})$ is required) for the number of distinct elements in $\sigma \circ \tau$ gives an answer to the original GHD instance. This reduction can be extended to multi-pass streaming algorithms in a natural way: when Bob is finished processing $\tau$, he sends the memory contents back to Alice, who begins processing $\sigma$ a second time. Generalizing, it is easy to see that a $p$-pass streaming algorithm gives a $(2 p-1)$-round communication protocol, where each message is the memory contents of the streaming algorithm. Accordingly, a lower bound on the length of the largest message of $(2 p-1)$ round protocols gives a space lower bound for the $p$-pass streaming algorithm.

Thus, the one-round linear lower bound by Indyk and Woodruff [10] yields the desired $\Omega\left(1 / \gamma^{2}\right)$ (one-pass) space lower bound for the streaming problem. Similarly, our new communication lower bounds imply $\Omega\left(1 /\left(\gamma^{2} p^{2} \log p\right)\right)$ space lower bounds for $p$ pass algorithms for the streaming problem. This improves on previous bounds for all $p=o\left(n^{1 / 4} / \sqrt{\log n}\right)$.

Organization of the paper. We start with some preliminaries in Section 2 including a discussion of the key measure concentration results that we will use, both for the sphere and for the Hamming cube, in Section 2.2 In Section 3 we prove our main result, while in Section 4 we give the simple combinatorial proof of the slightly weaker result mentioned above.

\section{Preliminaries}

Notation. For $x, y \in \mathbb{R}^{n}$, let $d(x, y):=\|x-y\|$ be the Euclidean distance between $x$ and $y$, and $x \cdot y$ their inner product. For $z \in \mathbb{R}$, define $\operatorname{sgn}(z):=0$ if $z \geq 0$, and $\operatorname{sgn}(z)=1$ otherwise. For a set $S \subseteq \mathbb{R}^{n}$, let $d(x, S)$ be the infimum over all $y \in S$ of $d(x, y)$. The unique rotationally-invariant probability distribution on the $n$-dimensional sphere $\mathbb{S}^{n-1}$ is the Haar measure, which we denote by $\nu$. When we say that a vector is taken from the uniform distribution over a measurable subset of the sphere, we will always mean that it is distributed according to the Haar measure, conditioned on being in that subset. 
Define the max-cost of a communication protocol to be the length of the longest single message sent during an execution of the protocol, for a worst-case input. We use $R_{\varepsilon}^{k}(f)$ to denote the minimal max-cost amongst all two-party, $k$-round, public-coin protocols that compute $f$ with error probability at most $\varepsilon$ on every input (here a "round" is one message).

\subsection{Problem Definition}

We will prove our lower bounds for the problem $\mathcal{G H} \mathcal{H}_{d, \gamma}$, where $d$ is an integer and $\gamma>0$. In this problem Alice receives a $d$-dimensional unit vector $x$, and Bob receives a $d$-dimensional unit vector $y$, with the promise that $|x \cdot y| \geq \gamma$. Alice and Bob should output $\operatorname{sgn}(x \cdot y)$.

We show that $\mathcal{G H} \mathcal{D}_{n, 1 / \sqrt{n}}$ has essentially the same randomized communication complexity as the problem GHD that we defined in the introduction. Generalizing that definition, for any $g>0$ define the problem $\mathrm{GHD}_{n, g}$, in which the input is formed of two $n$-bit strings $x$ and $y$, with the promise that $|\Delta(x, y)-n / 2| \geq g$, where $\Delta$ is the Hamming distance. Alice and Bob should output 0 if $\Delta(x, y)<n / 2$ and 1 otherwise.

The following proposition shows that for any $\sqrt{n} \leq g \leq n$, the problems $\mathrm{GHD}_{n, g}$ and $\mathcal{G H} \mathcal{D}_{d, \gamma}$ are essentially equivalent from the point of view of randomized communication complexity (with shared randomness) as long as $d \geq n$ and $\gamma=\Theta(g / n)$. It also shows that the randomized communication complexity of $\mathcal{G H} \mathcal{D}_{d, \gamma}$ is independent of the dimension $d$ of the input, as long as $d$ is large enough with respect to $\gamma$.

Proposition 1. For every $\varepsilon>0$, there is a constant $C_{0}=C_{0}(\varepsilon)$ such that for all integers $k, d \geq 0$ and $\sqrt{n} \leq g \leq n$, we have

$$
R_{2 \varepsilon}^{k}\left(\mathcal{G H} \mathcal{D}_{d, C_{0} g / n}\right) \leq R_{\varepsilon}^{k}\left(\operatorname{GHD}_{n, g}\right) \leq R_{\varepsilon}^{k}\left(\mathcal{G H} \mathcal{D}_{n, 2 g / n}\right)
$$

Proof. We begin with the right inequality. The idea is that a $\mathrm{GHD}_{n, g}$ protocol can be obtained by applying a given $\mathcal{G H D}$ protocol to a suitably transformed input. Let $x, y \in\{0,1\}^{n}$ be two inputs to $\mathrm{GHD}_{n, g}$. Define $\tilde{x}=\frac{1}{\sqrt{n}}\left((-1)^{x_{i}}\right)_{i \in[n]}$ and $\tilde{y}=$ $\frac{1}{\sqrt{n}}\left((-1)^{y_{i}}\right)_{i \in[n]}$. Then $\tilde{x}, \tilde{y} \in \mathbb{S}^{n-1}$. Moreover, $\tilde{x} \cdot \tilde{y}=1-2 \Delta(x, y) / n$. Therefore, if $\Delta(x, y) \geq n / 2+g$ then $\tilde{x} \cdot \tilde{y} \leq-2 g / n$, and if $\Delta(x, y) \leq n / 2-g$ then $\tilde{x} \cdot \tilde{y} \geq 2 g / n$. This proves $R_{\varepsilon}^{k}\left(\mathrm{GHD}_{n, g}\right) \leq R_{\varepsilon}^{k}\left(\mathcal{G H} \mathcal{D}_{n, 2 g / n}\right)$.

For the left inequality, let $x$ and $y$ be two unit vectors (in any dimension) such that $|x \cdot y| \geq \gamma$, where $\gamma=C_{0} g / n$. Note that since $g \geq \sqrt{n}$, we have $n=\Omega\left(\gamma^{-2}\right)$. Using shared randomness, Alice and Bob pick a sequence of vectors $w_{1}, \ldots, w_{n}$, each independently and uniformly drawn from the unit sphere. Define two $n$-bit strings $\tilde{x}=\left(\operatorname{sgn}\left(x \cdot w_{i}\right)\right)_{i \in[n]}$ and $\tilde{y}=\left(\operatorname{sgn}\left(y \cdot w_{i}\right)\right)_{i \in[n]}$. Let $\alpha=\cos ^{-1}(x \cdot y)$ be the angle between $x$ and $y$. Then a simple argument (used, e.g., by Goemans and Williamson [8]) shows that the probability that a random unit vector $w$ is $\operatorname{such}$ that $\operatorname{sgn}(x \cdot w) \neq$ $\operatorname{sgn}(y \cdot w)$ is exactly $\alpha / \pi$. This means that for each $i$, the bits $\tilde{x}_{i}$ and $\tilde{y}_{i}$ differ with probability $\frac{1}{\pi} \cos ^{-1}(x \cdot y)$, independently of the other bits of $\tilde{x}$ and $\tilde{y}$. The first few 
terms in the Taylor series expansion of $\cos ^{-1}$ are $\cos ^{-1}(z)=\frac{\pi}{2}-z-\frac{z^{3}}{6}+O\left(z^{5}\right)$. Hence, for each $i, \operatorname{Pr}_{w_{i}}\left(\tilde{x}_{i} \neq \tilde{y}_{i}\right)=1 / 2-\Theta(x \cdot y)$, and these events are independent for different $i$. Choosing $C_{0}$ sufficiently large, with probability at least $1-\varepsilon$, the Hamming distance between $\tilde{x}$ and $\tilde{y}$ is at most $n / 2-g$ if $x \cdot y \geq \gamma$, and it is at least $n / 2+g$ if $x \cdot y \leq-\gamma$.

\subsection{Concentration of Measure}

It is well known that the Haar measure $\nu$ on a high-dimensional sphere is tightly concentrated around the equator - around any equator, which makes it a fairly counterintuitive phenomenon. The original phrasing of this phenomenon, usually attributed to P. Lévy [14], goes by showing that among all subsets of the sphere, the one with the smallest "boundary" is the spherical cap $S_{\gamma}^{x}=\left\{y \in \mathbb{S}^{n-1}: x \cdot y \geq \gamma\right\}$. The following standard volume estimate will prove useful (see, e.g., [2], Lemma 2.2).

Fact 3. Let $x \in \mathbb{S}^{n-1}$ and $\gamma>0$. Then $\nu\left(S_{\gamma}^{x}\right) \leq e^{-\gamma^{2} n / 2}$.

Given a measurable set $A$, define its $t$-boundary $A_{t}:=\left\{x \in \mathbb{S}^{n-1}: d(x, A) \leq t\right\}$, for any $t>0$. At the core of our results will be the standard fact that, for any not-too-small set $A$, the set $A_{t}$ contains almost all the sphere, even for moderately small values of $t$.

Fact 4 (Concentration of measure on the sphere). For any measurable $A \subseteq \mathbb{S}^{n-1}$ and any $t>0$,

$$
\operatorname{Pr}(x \in A) \operatorname{Pr}\left(x \notin A_{t}\right) \leq 4 e^{-t^{2} n / 4},
$$

where the probabilities are taken according to the Haar measure on the sphere.

Proof. The usual measure concentration inequality for the sphere (Theorem 14.1.1 in [16]) says that for any set $B \subseteq \mathbb{S}^{n-1}$ of measure at least $1 / 2$ and any $t^{\prime}>0$,

$$
\operatorname{Pr}\left(x \notin B_{t^{\prime}}\right) \leq 2 e^{-\left(t^{\prime}\right)^{2} n / 2} .
$$

This suffices to prove the fact if $\operatorname{Pr}(x \in A) \geq 1 / 2$, so assume that $\operatorname{Pr}(x \in A)<1 / 2$. Let $t_{0}$ be such that $A_{t_{0}}$ has measure $1 / 2$; such a $t_{0}$ exists by continuity. Applying measure concentration to $B=A_{t_{0}}$ gives

$$
\operatorname{Pr}\left(x \notin A_{t^{\prime}+t_{0}}\right) \leq 2 e^{-\left(t^{\prime}\right)^{2} n / 2},
$$

for all $t^{\prime}>0$, while applying it to $B=\overline{A_{t_{0}}}$ yields

$$
\operatorname{Pr}\left(x \in A_{t_{0}-t^{\prime \prime}}\right) \leq \operatorname{Pr}\left(x \notin B_{t^{\prime \prime}}\right) \leq 2 e^{-\left(t^{\prime \prime}\right)^{2} n / 2}
$$

for all $t^{\prime \prime} \leq t_{0}$, since $A_{t_{0}-t^{\prime \prime}}$ is included in the complement of $\left(\overline{A_{t_{0}}}\right)_{t^{\prime \prime}}$. Taking $t^{\prime \prime}=t_{0}$ gives us $\operatorname{Pr}(x \in A) \leq 2 e^{-t_{0}^{2} n / 2}$. If $t \leq t_{0}$ then this suffices to prove the inequality. Otherwise, set $t^{\prime}:=t-t_{0}$ in (2) and $t^{\prime \prime}:=t_{0}$ in (3) and multiply the two inequalities to obtain the required bound, by using that $t_{0}^{2}+\left(t-t_{0}\right)^{2} \geq t^{2} / 2$ (which holds since $\left.2 t_{0}^{2}+t^{2} / 2-2 t t_{0}=\left(\sqrt{2} t_{0}-t / \sqrt{2}\right)^{2} \geq 0\right)$. 
Why the sphere? In Section 4 we give a proof of a slightly weaker lower bound than the one in our main result by using measure concentration facts on the Hamming cube only. We present those useful facts now, together with a brief discussion of the differences, in terms of concentration of measure phenomenon, between the Haar measure on the sphere and the uniform distribution over the hypercube. These differences point to the reasons why the proof of Section 4 gives an inferior bound.

On the Hamming cube, the analogous notion of spherical cap is the Hamming ball: let $T_{c}^{x}=\left\{y \in\{0,1\}^{n}: \Delta(x, y) \leq n / 2-c \sqrt{n}\right\}$ be the Hamming ball of radius $n / 2-c \sqrt{n}$ centered at $x$. The analogue of Fact 3 is given by the Chernoff bound:

Fact 5. For all $c>0$, we have $2^{-n}\left|T_{c}^{x}\right| \leq e^{-2 c^{2}}$.

A result similar to Lévy's, attributed to Harper [9], states that among all subsets (of the Hamming cube) of a given size, the ball is the one with the smallest boundary. Following a similar proof as for Fact 4 , one can get the following statement for the Hamming cube (see e.g. Corollary 4.4 in [3]):

Fact 6 (Concentration of measure on the Hamming cube). Let $A \subseteq\{0,1\}^{n}$ be any set, and define $A_{c}=\left\{x \in\{0,1\}^{n}: \exists y \in A, \Delta(x, y) \leq c \sqrt{n}\right\}$. Then

$$
\operatorname{Pr}(x \in A) \operatorname{Pr}\left(x \notin A_{c}\right) \leq e^{-c^{2}},
$$

where the probabilities are taken according to the uniform distribution on the Hamming cube.

To compare these two statements, embed the Hamming cube in the sphere by mapping $x \in\{0,1\}^{n}$ to the vector $v_{x}=\frac{1}{\sqrt{n}}\left((-1)^{x_{i}}\right)_{i \in[n]}$, so that two strings of Hamming distance $c \sqrt{n}$ are mapped to vectors with Euclidean distance $\sqrt{2 c} / n^{1 / 4}$. While on the sphere inequality (1) indicates that most points are at distance roughly $1 / \sqrt{n}$ from any set of measure half, if we are restricted to the Hamming cube then very few points are at a corresponding Hamming distance of 1 from, say, the set of all strings with fewer than $n / 21$ s, which has measure roughly $1 / 2$ in the cube. This difference is crucial: it indicates that the $n$-dimensional cube is too rough an approximation of the $n$-dimensional sphere for our purposes, perhaps explaining why our combinatorial bound in Section 4 yields a somewhat weaker dependence on the number of rounds.

\section{Main Result}

Our main result is the following.

Theorem 7. Let $0 \leq \varepsilon \leq 1 / 50$. There exist constants $C, C^{\prime}$ depending only on $\varepsilon$ such that the following holds for any $\gamma>0$ and any integers $n \geq \varepsilon^{2} /\left(4 \gamma^{2}\right)$ and $k \leq C^{\prime} /(\gamma \ln (1 / \gamma))$ : if $P$ is a randomized $\varepsilon$-error $k$-round communication protocol for $\mathcal{G} \mathcal{H} \mathcal{D}_{n, \gamma}$ then some message has length at least $\frac{C}{k^{2} \ln k} \cdot \frac{1}{\gamma^{2}}$ bits.

Using Proposition 1 we immediately get a lower bound for the Hamming cube version $\mathrm{GHD}=\mathrm{GHD}_{n, \sqrt{n}}$ : 
Corollary 1. Any $\varepsilon$-error $k$-round randomized protocolfor GHD communicates at least $\Omega\left(n /\left(k^{2} \ln k\right)\right)$ bits.

This follows from Theorem 7 when $k=o(\sqrt{n} / \log n)$. If $k$ is larger, then the bound stated in the Corollary is in fact weaker than the general $\Omega(\sqrt{n})$ lower bound which we sketched in the introduction.

\subsection{Proof Outline}

We now turn to the proof of Theorem 7 Let $\varepsilon, \gamma$ and $n$ be as in the statement of the theorem. Since lowering $n$ only makes the $\mathcal{G H} \mathcal{D}_{n, \gamma}$ problem easier, for the rest of this section we assume that $n:=\varepsilon^{2} /\left(4 \gamma^{2}\right)$ is fixed, and for simplicity of notation we write $\mathcal{G H} \mathcal{D}_{\gamma}$ for $\mathcal{G H} \mathcal{D}_{n, \gamma}$.

Measurability. Before proceeding with the proof, we first need to handle a small technicality arising from the continuous nature of the input space: namely, that the distributional protocol might make decisions based on subsets of the input space that are not measurable. To make sure that this does not happen, set $\delta=\gamma / 6$ and consider players Alice and Bob who first round their inputs to the closest vector in a fixed $\delta$-net, and then proceed with an $\varepsilon$-error protocol for $\mathcal{G H} \mathcal{D}_{\gamma / 2}$. Since by definition rounding to the $\delta$-net moves any vector a distance at most $\delta$, the rounding will affect the inner product $x \cdot y$ by at most $2 \delta+\delta^{2} \leq \gamma / 2$. As a result, Alice and Bob will succeed with probability $1-\varepsilon$ provided they are given valid inputs to $\mathcal{G H} \mathcal{D}_{\gamma}$. Hence any randomized $\varepsilon$-error protocol for $\mathcal{G H} \mathcal{D}_{\gamma / 2}$ can be transformed into a randomized $\varepsilon$-error protocol for $\mathcal{G H} \mathcal{D}_{\gamma}$ with the same communication, but which initially rounds its inputs to a discrete set. We prove a lower bound on the latter type of protocol. This will ensure that all sets encountered in the proof are measurable.

Distributional complexity. By Yao's principle it suffices to lower-bound the distributional complexity, i.e., to analyze deterministic protocols that are correct with probability $1-\varepsilon$ under some input distribution. As our input distribution for $\mathcal{G H} \mathcal{D}_{\gamma}$ we take the distribution that is uniform over the inputs satisfying the promise $|x \cdot y| \geq \gamma$. Given our choice of $n$, Claim 8 below guarantees that the $\nu \times \nu$-measure of non-promise inputs is at most $\varepsilon$. Hence it will suffice to lower-bound the distributional complexity of protocols making error at most $2 \varepsilon$ under the distribution $\nu \times \nu$. We define an $\varepsilon$-protocol to be a deterministic communication protocol for $\mathcal{G H} \mathcal{D}_{n, \gamma}$ whose error under the distribution $\nu \times \nu$ is at most $\varepsilon$, where we say that a protocol $P$ makes an error if $P(x, y) \neq \operatorname{sgn}(x, y)$.

We prove a lower bound on the maximum length of a message sent by any $\varepsilon$-protocol, via round elimination. The main reduction step is given by the following technical lemma:

Lemma 1 (Round Elimination on the sphere). Let $\varepsilon, \gamma>0, n=\varepsilon^{2} /\left(4 \gamma^{2}\right)$, and $1 \leq \kappa \leq k$. Assume there is a $\kappa$-round $\varepsilon$-protocol $P$ such that the first message has length bounded as $c_{1} \leq C_{1} \frac{n}{k^{2} \ln k}-7 \ln (2 k)$ where $C_{1}$ is a universal constant. Then there is a $(\kappa-1)$-round $\varepsilon^{\prime}$-protocol $Q$ (obtained by eliminating the first message of $P$ ), where $\varepsilon^{\prime} \leq\left(1+\frac{1}{k}\right) \varepsilon+\frac{1}{16 k}$. 
Before proving this lemma in Section 3.2, we show how it implies Theorem 7 .

Proof (of Theorem 7). We will show that in any $k$-round $(2 \varepsilon)$-protocol, there is a message sent of length at least $C_{1} n /\left(k^{2} \ln k\right)-7 \ln (2 k)$. The discussion in the "Distributional complexity" paragraph above shows this suffices to prove the theorem, by setting $C=C_{1} \varepsilon^{2} / 8$, and choosing $C^{\prime}$ small enough so that the bound on $k$ in the statement of the theorem implies that $7 \ln (2 k)<C_{1} n /\left(2 k^{2} \ln k\right)$.

Let $P$ be a $k$-round $(2 \varepsilon)$-protocol, and assume for contradiction that each round of communication uses at most $C_{1} n /\left(k^{2} \ln k\right)-7 \ln (2 k)$ bits. Solving the recurrence $\varepsilon_{\kappa}=(1+1 / k) \varepsilon_{\kappa-1}+1 /(16 k), \varepsilon_{0}=2 \varepsilon$ gives $\varepsilon_{\kappa}=(1+1 / k)^{\kappa}(2 \varepsilon+1 / 16)-1 / 16$, so that applying Lemma $1 k$ times leads to a 0 -round protocol for $\mathcal{G H \mathcal { H }}$, that errs with probability at most $\varepsilon^{\prime} \leq e(2 \varepsilon+1 / 16)-1 / 16 \leq 1 / 4$ over the input distribution $\nu \times \nu$. We have reached a contradiction: such a protocol needs communication and hence cannot be 0 -round. Hence $P$ must send a message of length at least $C_{1} n /\left(k^{2} \ln k\right)-7 \ln (2 k)$.

\subsection{The Main Reduction Step}

Proof (of Lemma 1). Let $P(x, y)$ denote the output of the protocol on input $x, y$. Define $x \in \mathbb{S}^{n-1}$ to be good if $\operatorname{Pr}_{\nu \times \nu}(P(x, y)$ errs $\mid x) \leq(1+1 / k) \varepsilon$. By Markov's inequality, at least a $1 /(k+1)$-fraction of $x$ (distributed according to $\nu$ ) are good. For a given message $m$, let $A_{m}$ be the set of all good $x$ on which Alice sends $m$ as her first message. The sets $A_{m}$, over all messages $m \in\{0,1\}^{c_{1}}$, form a partition of the set of good $x$. Define $m_{1}:=\operatorname{argmax}_{m} \nu\left(A_{m}\right)$ and let $A:=A_{m_{1}}$. We then have $\nu(A) \geq \frac{1}{k+1} 2^{-c_{1}} \geq$ $e^{-c_{1}-\ln (k+1)}$.

We now define protocol $Q$. Alice receives an input $\tilde{x}$, Bob receives $\tilde{y}$, both distributed according to $\nu$. Alice computes the point $x \in A$ that is closest to $\tilde{x}$, and Bob sets $y:=\tilde{y}$. They run protocol $P(x, y)$ without Alice sending the first message, so Bob starts and proceeds as if he received $m_{1}$ from Alice.

To prove the lemma, it suffices to bound the error probability $\varepsilon^{\prime}$ of $Q$ with input $\tilde{x}, \tilde{y}$ distributed according to $\nu \times \nu$. Define $d_{1}=2 \sqrt{\frac{c_{1}+6 \ln (2 k)+2}{n}}$. We consider the following bad events:

- $\mathrm{BAD}_{1}: d(\tilde{x}, A)>d_{1}$,

$-\mathrm{BAD}_{2}: P(x, y) \neq \operatorname{sgn}(x \cdot y)$,

- $\mathrm{BAD}_{3}: d(\tilde{x}, A) \leq d_{1}$ but $\operatorname{sgn}(x \cdot y) \neq \operatorname{sgn}(\tilde{x} \cdot \tilde{y})$.

If none of those events occurs, then protocol $P$ outputs the correct answer. We bound each of them separately, and will conclude by upper bounding $\varepsilon^{\prime}$ with a union bound.

The first bad event can be easily bounded using the measure concentration inequality from Fact 4 Since $\tilde{x}$ is uniformly distributed in $\mathbb{S}^{n-1}$ and $\operatorname{Pr}(A) \geq e^{-c_{1}-\ln (k+1)}$, we get

$$
\operatorname{Pr}\left(\mathrm{BAD}_{1}\right) \leq 4 e^{-d_{1}^{2} n / 4+c_{1}+\ln (k+1)} \leq 4 e^{-5 \ln (2 k)-2} \leq \frac{1}{32 k} .
$$

The second bad event has probability bounded by $(1+1 / k) \varepsilon$ by the goodness of $x$. Now consider event $\mathrm{BAD}_{3}$. Without loss of generality, we may assume that $\tilde{x} \cdot \tilde{y}=\tilde{x} \cdot y>0$ 
but $x \cdot y<0$ (the other case is treated symmetrically). In order to bound $\mathrm{BAD}_{3}$, we will use two claims. The first shows that the probability that $\tilde{x} \cdot y$ is close to 0 for a random $\tilde{x}$ and $y$ is small. The second uses measure concentration to show that, if $\tilde{x} \cdot y$ is not too close to 0 , then moving $\tilde{x}$ to the nearby $x$ is unlikely to change the sign of the inner product.

Claim 8. Let $x, y$ be distributed according to $\nu$. For any real $\alpha \geq 0$, we have

$$
\operatorname{Pr}(0 \leq x \cdot y \leq \alpha) \leq \alpha \sqrt{n}
$$

Proof. With $\omega_{n}$ the volume of the $n$-dimensional Euclidean unit ball, we write (see e.g. [4], Lemma 5.1)

$$
\operatorname{Pr}(0 \leq x \cdot y \leq \alpha)=\frac{(n-1) \omega_{n-1}}{n \omega_{n}} \int_{0}^{\alpha}\left(1-t^{2}\right)^{\frac{n-3}{2}} \mathrm{dt} \leq \alpha \sqrt{n},
$$

where we used $\frac{\omega_{n-1}}{\omega_{n}}<\sqrt{\frac{n+1}{2 \pi}}<\sqrt{n}$.

Claim 9. Let $x, \tilde{x}$ be two fixed unit vectors at distance $\|x-\tilde{x}\|=d \in\left[0, d_{1}\right]$, and $0<\alpha \leq 1 /(4 \sqrt{n})$. Let $y$ be taken according to $\nu$. Then

$$
\operatorname{Pr}(\tilde{x} \cdot y \geq \alpha \wedge x \cdot y<0) \leq e^{-\alpha^{2} n /\left(8 d_{1}^{2}\right)} .
$$

Proof. Note that $x \cdot \tilde{x}=1-\|x-\tilde{x}\|^{2} / 2=1-d^{2} / 2$. Since the statement of the lemma is rotationally-invariant, we may assume without loss of generality that

$$
\begin{aligned}
& \tilde{x}=(1,0,0 \ldots, 0), \\
& x=\left(1-d^{2} / 2,-\sqrt{d^{2}-d^{4} / 4}, 0, \ldots, 0\right), \\
& y=\left(y_{1}, y_{2}, y_{3}, \ldots, y_{n}\right) .
\end{aligned}
$$

Therefore, $y_{1} \geq \alpha$ when $\tilde{x} \cdot y \geq \alpha$. Note that

$$
x \cdot y=x_{1} y_{1}+x_{2} y_{2} \geq\left(1-d^{2} / 2\right) \alpha-\sqrt{d^{2}-d^{4} / 4} y_{2} .
$$

Hence the event $\tilde{x} \cdot y \geq \alpha \wedge x \cdot y<0$ implies

$$
y_{2}>\frac{\left(1-d^{2} / 2\right) \alpha}{\sqrt{d^{2}-d^{4} / 4}} \geq \frac{\alpha}{2 d},
$$

where we used the fact that $d \leq d_{1} \leq 1$, given our assumption on $c_{1}$. By Fact 3 the probability that, when $y$ is sampled from $\nu, y_{2}$ is larger than $\alpha /(2 d)$ is at most $e^{-\alpha^{2} n /\left(8 d^{2}\right)}$. Hence the probability that both $\tilde{x} \cdot y \geq \alpha$ and $x \cdot y<0$ happen is at most as much.

Setting $\alpha=1 /(128 k \sqrt{n})$, by Claim 8 we find that the probability that $0 \leq \tilde{x} \cdot y \leq \alpha$ is at most $1 /(128 k)$. Furthermore, the probability that $\tilde{x} \cdot y \geq \alpha$ and $x \cdot y<0$ is at most $\exp \left(-\frac{n}{2^{19} k^{2}\left(c_{1}+6 \ln (2 k)+2\right)}\right)$ by Claim 9 . This bound is less than $1 /(128 k)$ given 
our assumption on $c_{1}$, provided $C_{1}$ is a small enough constant. Putting both bounds together, we see that

$$
\operatorname{Pr}(\tilde{x} \cdot y \geq 0 \wedge x \cdot y<0)<1 /(64 k) .
$$

The event that $\tilde{x} \cdot y<0$ but $x \cdot y \geq 0$ is bounded by $1 /(64 k)$ in a similar manner. Hence, $\operatorname{Pr}\left(\mathrm{BAD}_{3}\right)<1 /(32 k)$. Taking the union bound over all three bad events concludes the proof of the lemma.

\section{A Simple Combinatorial Proof}

In this section we present a combinatorial proof of the following:

Theorem 10. Let $0 \leq \varepsilon \leq 1 / 50$. There exists a constant $C^{\prime \prime}$ depending on $\varepsilon$ only, such that the following holds for any $g \leq C^{\prime \prime} \sqrt{n}$ and $k \leq n^{1 / 4} /(1024 \log n)$ : if $P$ is a randomized $\varepsilon$-error $k$-round communication protocol for $\mathrm{GHD}_{n, g}$ then some message has length at least $\frac{n}{(512 k)^{4} \log ^{2} k}$ bits.

Even though this is a weaker result than Theorem 7 its proof is simpler and is based on concentration of measure in the Hamming cube rather than on the sphere (we refer to Section 2.2 for a high-level comparison of the two proofs). Interestingly, the dependence on the number of rounds that we obtain is quadratically worse than that of the proof using concentration on the sphere. We do not know if this can be improved using the same technique.

We proceed as in Section 3.1, observing that it suffices to lower-bound the distributional complexity of $\mathrm{GHD}_{n, g}$ under a distribution uniform over the inputs satisfying the promise $|\Delta(x, y)-n / 2| \geq g$. In fact, as we did before, by taking $C^{\prime \prime}$ small enough we can guarantee that the number of non-promise inputs is at most $\varepsilon 2^{n}$. Hence it will suffice to lower-bound the distributional complexity of protocols making error at most $2 \varepsilon$ under the uniform input distribution. We define an $\varepsilon$-protocol to be a deterministic communication protocol for GHD whose distributional error under the uniform distribution is at most $\varepsilon$. The following is the analogue of Lemma1 from which the proof of Theorem 10 follows as in Section 3.1 .

Lemma 2 (Round Elimination on the Hamming cube). Let $\varepsilon>0$ and $\kappa$, $k$ be two integers such that $k \geq 128$ and $1 \leq \kappa \leq k \leq n^{1 / 4} /(1024 \log n)$. Assume that there is a $\kappa$-round $\varepsilon$-protocol $P$ such that the first message has length bounded by $c_{1} \leq n /\left((512 k)^{4} \log ^{2} k\right)$. Then there exists a $(\kappa-1)$-round $\varepsilon^{\prime}$-protocol $Q$ (obtained by eliminating the first message of $P$ ) where $\varepsilon^{\prime} \leq\left(1+\frac{1}{k}\right) \varepsilon+\frac{1}{16 k}$.

Proof. Define $x \in\{0,1\}^{n}$ to be good if $\operatorname{Pr}(P(x, y)$ errs $\mid x) \leq(1+1 / k) \varepsilon$. By Markov's inequality, at least a $1 /(k+1)$-fraction of $x \in\{0,1\}^{n}$ are good. For a given message $m$, let $A_{m}:=\{\operatorname{good} x$ : Alice sends $m$ given $x\}$. The sets $A_{m}$, over all messages $m \in$ $\{0,1\}^{c_{1}}$, together form a partition of the set of $\operatorname{good} x$. Define $m_{1}:=\operatorname{argmax}_{m}\left|A_{m}\right|$, and let $A:=A_{m_{1}}$. By the pigeonhole principle, we have $|A| \geq \frac{1}{k+1} 2^{n-c_{1}}$.

We now define protocol $Q$. Alice receives an input $\tilde{x}$, Bob receives $\tilde{y}$, uniformly distributed. Alice computes the string $x \in A$ that is closest to $\tilde{x}$ in Hamming distance, 
and Bob sets $y:=\tilde{y}$. They run protocol $P(x, y)$ without Alice sending the first message, so Bob starts and proceeds as if he received the fixed message $m_{1}$ from Alice.

To prove the lemma, it suffices to bound the error probability $\varepsilon^{\prime}$ of $Q$ under the uniform distribution. Define $d_{1}:=9 \sqrt{n} /\left((1024 k)^{2} \log k\right)$. As in the proof of Lemma1, we consider the following bad events:

- $\mathrm{BAD}_{1}: \Delta(x, \tilde{x})>d_{1} \sqrt{n}$,

- $\mathrm{BAD}_{2}: P(x, y) \neq \operatorname{GHD}(x, y)$,

- $\mathrm{BAD}_{3}: \Delta(x, \tilde{x}) \leq d_{1} \sqrt{n}$ but $\operatorname{GHD}(\tilde{x}, y) \neq \mathrm{GHD}(x, y)$.

If none of those events occurs, then protocol $P$ outputs the correct answer. We bound each of them separately, and will conclude by a union bound. $\mathrm{BAD}_{1}$ is easily bounded using Fact 6, which implies

$$
\operatorname{Pr}\left(\tilde{x} \notin A_{d_{1}}\right) \leq e^{-81 n /\left((1024 k)^{4} \log ^{2} k\right)} 2^{c_{1}+\log (k+1)} \leq \frac{2}{k^{2}} \leq \frac{1}{32 k},
$$

given our assumptions on $c_{1}$ and $k$. The second bad event is bounded by $(1+1 / k) \varepsilon$, by definition of $A$.

We now turn to $\operatorname{BAD}_{3}$. The event that $\operatorname{GHD}(\tilde{x}, y) \neq \operatorname{GHD}(x, y)$ only depends on the relative distances between $x, \tilde{x}$, and $y$, so we may apply a shift to assume that $x=(0, \ldots, 0)$. Without loss of generality, we assume that $\Delta(\tilde{x}, y)>n / 2$ and $|y|<$ $n / 2$ (the error bound when $\Delta(\tilde{x}, y)<n / 2$ and $|y|>n / 2$ is proved in a symmetric manner). Note that, since $y$ is uniformly random (subject to $|y|<n / 2$ ), by a standard head estimate for the binomial distribution with probability at least $1-1 /(128 k)$ we have $|y| \leq n / 2-\sqrt{n} /(128 k)$ (this is analoguous to the estimate from Claim 8 that we used in the continuous setting). Hence we may assume that this holds with an additive loss of at most $1 /(128 k)$ in the error. Now

$$
\Delta(\tilde{x}, y)>n / 2 \Longleftrightarrow|\tilde{x}|+|y|-2|\tilde{x} \cap y|>n / 2 \Longleftrightarrow|\tilde{x} \cap y|<\frac{|\tilde{x}|+|y|-n / 2}{2} .
$$

It is clear that the worst case in this statement is for $|y|=n / 2-\sqrt{n} /(128 k)$ and $|\tilde{x}|=\Delta(x, \tilde{x})=d_{1} \sqrt{n}$. By symmetry, the probability that this event happens is the same as if we fix any $y$ of the correct weight, and $\tilde{x}$ is a random string of weight $d_{1} \sqrt{n}$. The expected intersection size is $|y||\tilde{x}| / n=|\tilde{x}| / 2-d_{1} /(128 k)$, and so by Hoeffding's inequality (see e.g. the bound on the tail of the hypergeometric distribution given in [7]), for $a=\sqrt{n} /(256 k)-d_{1} /(128 k)$, we have

$$
\operatorname{Pr}\left(|\tilde{x} \cap y| \leq \frac{|\tilde{x}|+|y|-n / 2}{2}\right)=\operatorname{Pr}(|\tilde{x} \cap y| \leq \mathbb{E}[|\tilde{x} \cap y|]-a) \leq e^{-2 a^{2} /\left(d_{1} \sqrt{n}\right)} .
$$

Given our choice of $d_{1}$ we have $a \geq 3 \sqrt{n} /(4 \cdot 256 k)$, and hence the upper bound is at most $1 / k^{2} \leq 1 /(128 k)$, given our assumption on $k$. Applying the union bound over all bad events then yields the lemma.

Acknowledgments. We thank Ishay Haviv for discussions during the early stages of this work. 


\section{References}

1. Ajtai, M.: A lower bound for finding predecessors in Yao's cell probe model. Combinatorica 8, 235-247 (1988)

2. Ball, K.: An elementary introduction to modern convex geometry. Flavors of Geometry 31 (1997)

3. Barvinok, A.: Lecture notes on measure concentration (2005), http://www.math.1sa.umich.edu/ barvinok/total710.pdf

4. Brieden, A., Gritzmann, P., Kannan, R., Klee, V., Lovász, L., Simonovits, M.: Approximation of diameters: Randomization doesn't help. In: Proceedings of 39th IEEE Symposium on Foundations of Computer Science (FOCS 1998), pp. 244-251 (1998)

5. Brody, J., Chakrabarti, A.: A multi-round communication lower bound for Gap Hamming and some consequences. In: Proceedings of 24th IEEE Conference on Computational Complexity (CCC 2009), pp. 358-368 (2009)

6. Chakrabarti, A., Regev, O.: Tight lower bound for the Gap Hamming problem. Personal Communication (2009)

7. Chvátal, V.: The tail of the hypergeometric distribution. Discrete Mathematics 25(3), 285287 (1979)

8. Goemans, M., Williamson, D.: Improved approximation algorithms for maximum cut and satisfiability problems using semidefinite programming. Journal of the ACM 42, 1115-1145 (1995)

9. Harper, L.: Optimal numbering and isoperimetric problems on graphs. Journal of Combinatorial Theory 1, 385-393 (1966)

10. Indyk, P., Woodruff, D.: Tight lower bounds for the distinct elements problem. In: Proceedings of 44th IEEE Symposium on Foundations of Computer Science (FOCS 2003), pp. 283289 (2003)

11. Jayram, T.S., Kumar, R., Sivakumar, D.: The one-way communication complexity of Hamming distance. Theory of Computing 4(1), 129-135 (2008)

12. Kushilevitz, E., Nisan, N.: Communication Complexity. Cambridge University Press, Cambridge (1997)

13. Lee, T.,, S.: Disjointness is hard in the multi-party number-on-the-forehead model. In: Proceedings of 23rd IEEE Conference on Computational Complexity (CCC 2008), pp. 81-91 (2008)

14. Lévy, P.: Problèmes concrets d'analyse fonctionnelle. Gauthier-Villars (1951)

15. Linial, N., Shraibman, A.: Lower bounds in communication complexity based on factorization norms. In: Proceedings of 39th ACM Symposium on the Theory of Computing (STOC 2007), pp. 699-708 (2007)

16. Matoušek, J.: Lectures on Discrete Geometry. Springer, Heidelberg (2002)

17. Miltersen, P., Nisan, N., Safra, S., Wigderson, A.: On data structures and asymmetric communication complexity. J. Comput. Syst. Sci. 57(1), 37-49 (1998); preliminary version in Proceedings of 27th ACM Symposium on the Theory of Computing (STOC 1995), pp. 103111 (1995)

18. Razborov, A.: Quantum communication complexity of symmetric predicates. Izvestiya of the Russian Academy of Science, Mathematics 67, 0204025 (2002)

19. Sherstov, A.: The pattern matrix method for lower bounds on quantum communication. In: Proceedings of 40th ACM Symposium on the Theory of Computing (STOC 2008), pp. 85-94 (2008)

20. Woodruff, D.: Optimal space lower bounds for all frequency moments. In: Proceedings of 15th ACM-SIAM Symposium on Discrete Algorithms (SODA 2004), pp. 167-175 (2004) 